\title{
Factors associated with lower knowledge of HIV and STI transmission, testing and treatment among MSM in Ireland: findings from the MSM Internet Survey Ireland (MISI) 2015
}

\author{
Cian Carey (D) , ${ }^{1,2}$ Kate O'Donnell, ${ }^{3}$ Martin Davoren, ${ }^{2,4}$ Mick Quinlan, ${ }^{5}$ Derval Igoe, ${ }^{3}$ \\ Peter Barrett ${ }^{2,6}$
}

- Additional material is published online only. To view, please visit the journal online (http://dx.doi.org/10.1136/ sextrans-2020-054469).

'School of Medicine, University College Cork, Cork, Ireland ${ }^{2}$ School of Public Health, University College Cork, Cork, Ireland

${ }^{3} \mathrm{HSE}$ Health Protection Surveillance Centre, Dublin, Ireland

${ }^{4}$ Sexual Health Centre, University College Cork, Cork, Ireland

${ }^{5}$ Gay Health Network, Dublin, Ireland

${ }^{6}$ Department of Public Health HSE South, St. Finbarr's Hospital, Cork, Ireland

\section{Correspondence to}

Dr Peter Barrett, School of Public Health, University College Cork College of Medicine and Health, Cork, Ireland; peterbarrett1@ hotmail.com

Received 4 February 2020 Revised 1 September 2020

Accepted 3 October 2020

Published Online First

26 October 2020

Check for updates

(C) Author(s) (or their employer(s)) 2021. No commercial re-use. See rights and permissions. Published by BMJ.

To cite: Carey $\mathrm{C}$

O'Donnell K, Davoren M,

et al. Sex Transm Infect

2021:97:351-356.

\section{ABSTRACT}

Background Poor knowledge regarding STI and HIV transmission, testing and treatment among men who have sex with men (MSM) may be contributing to their disproportionate burden of STIs. However, factors which predispose MSM to having this low knowledge are less understood.

Aim The aim of this study was to identify factors associated with having lower knowledge of HIV and STI transmission, testing and treatment among MSM. Methods The MSM Internet Survey Ireland 2015 was a self-completed online national survey available to MSM living in Ireland. Thirteen factual statements were used to assess participants' knowledge of HIV and STI transmission, testing and treatment. Respondents were defined as having 'lower knowledge' if they indicated prior knowledge of fewer than 11 true statements. Multivariable-adjusted logistic regression was used to identify factors associated with having lower knowledge. Results 3090 MSM completed the survey, of whom 2905 (94\%) were included in this study. Thirty-six per cent ( $n=1055)$ had lower knowledge of HIV and STI transmission, testing and treatment. The factors associated with having lower knowledge included being $18-24$ years of age (vs $>40$ years; adjusted OR (aOR) $1.98,95 \% \mathrm{Cl} 1.50$ to 2.61 ), born in Ireland (vs outside Ireland; aOR $1.62,95 \% \mathrm{Cl} 1.25$ to 2.10 ) and being out to none of their contacts (vs out to most/all; aOR 1.69, $95 \% \mathrm{Cl} 1.20$ to 2.37). Knowledge was also lower among those who never tested for HIV (vs tested negative within last 12 months; aOR $2.32,95 \%$ Cl 1.88 to 2.86 ) and among MSM who never visited an MSM-specific health promotion website (vs visited website; aOR 1.81, 95\% Cl 1.45 to 2.25$)$.

Conclusion A range of demographic factors, sexual health variables and use of MSM-specific sexual health promotion materials are associated with low knowledge regarding HIV and STI transmission, testing and treatment among MSM in Ireland. Engagement with the main national MSM-specific sexual health promotion website was associated with higher knowledge levels.

\section{BACKGROUND}

STIs disproportionately affect men who have sex with men (MSM). ${ }^{1}$ Although MSM only represent approximately $6 \%$ of the Irish male population, they accounted for $49 \%$ of HIV, $86 \%$ of early infectious syphilis and $64 \%$ of gonorrhoea diagnoses in the overall population in Ireland in $2018 .^{23}$ This follows a similar trend throughout Europe and in the USA, where in recent years MSM have accounted for $50 \%$ and $67 \%$ of newly diagnosed HIV cases, respectively. ${ }^{45}$

Previous research highlights that MSM are more likely to experience an earlier sexual debut, are more likely to have multiple concurrent sexual partners and report longer cumulative lifetime periods of new partner acquisition when compared with heterosexual men. ${ }^{67}$ These behavioural issues, coupled with the fact that the risk of HIV transmission from condomless anal intercourse (CAI) is higher than unprotected vaginal sex, partially explain the higher rates of HIV in this group. ${ }^{8}$ In addition, many sociostructural issues, including the stigmatisation of the MSM population, have been shown to increase MSM vulnerability for HIV acquisition. ${ }^{910}$

Improved knowledge of HIV and STI transmission pathways is associated with increased awareness of preventive interventions and reduced spread of HIV/STI among sexual partners. ${ }^{11}$ Although knowledge is only one component of health literacy, previous research has highlighted the positive association between HIV-related knowledge and overall health literacy levels. ${ }^{12}$ It remains relevant and timely to conduct dedicated research on knowledge of HIV/STI among MSM, given the advances made in prevention and treatment in recent years. Younger MSM are growing up in a post-AIDS era, with ever-increasing availability of both treatment as prevention and pre-exposure prophylaxis ( $\mathrm{PrEP})$ for HIV, but with a growing threat from treatmentresistant STIs. Public health interventions, which can address low levels of HIV and STI-related knowledge at an individual level, have the potential to confer considerable benefits. A previous systematic review reported that MSM who received online educational interventions reported improved knowledge levels and had lower incidence of CAI with non-steady partners subsequently. ${ }^{13}$

Few studies from the European context have been able to clearly identify which subgroups of MSM 
have lower knowledge levels, and existing studies have been limited by relatively small sample sizes or by assessing participants' knowledge of HIV alone, without considering other STIs. ${ }^{14} 15$ The only previous study in an Irish context focused on MSM and heterosexuals together, and while it highlighted knowledge deficits regarding HIV transmission, there were too few MSM respondents for a dedicated analysis. ${ }^{16}$

Using data from the MSM Internet Survey Ireland (MISI 2015), the aims of this study were to compare MSM who had lower levels of knowledge of HIV and STI transmission, testing and treatment with MSM who did not, and to identify factors which may predispose them to having lower knowledge levels.

\section{METHODS}

\section{Study survey design}

We used data from the MISI 2015, which were an anonymous, cross-sectional survey of MSM living in Ireland. The survey was accessible online from 1 March to 31 May 2015 and was conducted prior to the introduction of PrEP in Ireland in 2017. The survey, which included 112 questions, was only available in English and took approximately $14 \mathrm{~min}$ to complete. Questions in MISI 2015 were derived from previous surveys, including the UK Gay Men's Sex Survey 2014 and the European MSM Internet Survey 2010. Certain questions were adapted and added to make the survey relevant to an Irish setting. Further details regarding the survey methodology are available in the MISI 2015 survey report. ${ }^{17}$

\section{Study population and sample}

The inclusion criteria for MISI 2015 were as follows: living in Ireland, identifying as a man or trans man, 18 years or older, sexually attracted to men and/or previously had sex with men or expected to in the future.

Survey participants were recruited via advertising on lesbian, gay, bisexual and transgender (LGBT+), STI and health promotion websites, through social media campaigns and through promotional card distribution at gay social and community venues. A launch party was held to initially promote the survey, with subsequent advertisements placed in national media and banner advertisements and emails used to target subscribers on gay networking apps. The majority (61\%) of men accessed the survey via the MISI website directly, with the breakdown of visitor numbers from recruiting websites available in the full survey report. ${ }^{17}$ Further detailed information on the survey promotion has been described elsewhere. ${ }^{18} 19$

\section{Survey measurement}

Lower knowledge of HIV and STI transmission, testing and treatment was our primary outcome of interest. Knowledge was assessed in this survey using 13 factually correct statements, which are presented in box 1 . These statements were used to examine men's overall HIV literacy and were not used as an examination of their personal HIV risk reduction strategies. The first seven statements related to an individual's prior knowledge of HIV testing and treatment, while the other six examined an individual's knowledge of transmission of HIV and STIs. After reading each statement, participants were asked to specify their prior knowledge by selecting one of five responses: I knew this already/I was not sure about this/I did not know this already/I do not understand this/I do not believe this (online supplemental table 1).

Using participants' responses for each of these statements, we derived a binary variable for each statement of 'knew' (I knew
Box 1 Statements used to assess men who have sex with men knowledge of HIV/STI transmission, testing and treatment

Statements regarding knowledge of HIV, testing and treatment (used in primary analysis only)

1. AIDS is caused by a virus called HIV.

2. You cannot be confident about whether someone has HIV or not from their appearance.

3. There is a medical test that can show whether or not you have HIV.

4. If someone becomes infected with HIV, it may take several weeks before it can be detected in a test.

5. There is currently no cure for HIV infection.

6. HIV infection can be controlled with medicines so that its impact on health is much less.

7. Effective treatment of HIV infection reduces the risk of HIV being transmitted.

Statements regarding HIV/STI transmission (used in primary ans secondary analyses)

1. HIV cannot be passed during kissing, including deep kissing, because saliva does not transmit HIV.

2. People can pick up HIV through their penis while being 'active' in unprotected intercourse (fucking) with an infected partner even if they don't ejaculate.

3. People can pick up HIV through their rectum while being passive in unprotected intercourse (being fucked) with an infected partner.

4. Even without ejaculation, oral sex (sucking and being sucked) carries a risk of infection with syphilis or gonorrhoea.

5. When HIV infected and uninfected people have sex together, the chances of HIV being passed on are greater if either partner has another STI.

6. Most STIs can be passed on more easily than HIV.

this already response) or 'did not know' (any of the other four responses). We next calculated the total number of statements each participant knew out of 13. Finally, we developed a binary variable of 'lower knowledge' if the respondent knew 10 statements or fewer out of 13 . Eleven or more out of 13 was defined as 'higher knowledge'. As MISI 2015 was a new survey, this cutoff was chosen to ensure an adequate number of respondents in each group and to reduce the risk of a type II error. This binary lower knowledge variable was then included as the dependent variable in the primary analysis. A dichotomous outcome variable was chosen (lower vs higher knowledge) for analysis due to skewed distribution in the numbers of men who knew each number of statements (online supplemental table 2). The use of a continuous outcome would have required a log (or other) transformation to be applied, and this may have impacted on the ability for clear public health messages to be drawn from the results.

We undertook a secondary analysis which focused on participants' knowledge of HIV/STI transmission (excluding statements related to testing or treatment). A previous cross-sectional study demonstrated poor knowledge of HIV/STI transmission among the general Irish population, and thus, this warranted inclusion as a separate analysis. ${ }^{16}$ This was performed using the six HIV and STI transmission statements that were included among the original 13 statements as discussed previously (last 6 statements in box 1). We again derived a new binary variable for lower versus higher knowledge, where lower knowledge 
for this analysis was four or fewer out of six, with five or more correct answers deemed to indicate higher knowledge of HIV/ STI transmission.

\section{Data analysis}

Primary analysis was restricted to respondents who answered all 13 questions about HIV/STI transmission, testing and treatment.

Associations between a range of demographic factors, exposure to health promotional materials and other variables pertaining to sexual health and orientation with overall knowledge levels were investigated. These covariates were selected a priori based on previous studies, which suggested associations with overall knowledge of HIV and risky sexual behaviours. ${ }^{19} 20$

The included demographic and sexual health/orientation factors can be seen in box 1 and are described in the supplement.

Furthermore, we included two binary covariates in the models relating to men's exposure to specific health promotion materials. These materials included the national governmentsupported MSM-specific sexual health promotion website ( www.man2man.ie) and four infographics, which had been previously developed by community partners to provide sexual health advice for MSM in Ireland (online supplemental file 1). The infographics covariate was derived as having seen at least one of the four infographics or having seen none of them previously. Similarly, we included a covariate for having visited the MSMspecific website or not.

Univariable analysis was performed between each of these covariates and lower knowledge among MSM. ORs were calculated with 95\% CIs and two-sided significance set at $\mathrm{p}<0.05$. We considered the overall proportion of MSM with lower knowledge to be the most relevant outcome, rather than count data, and thus, we used logistic regression rather than log-linear models (eg, Poisson regression). Subsequently, multivariableadjusted logistic regression analysis was undertaken, with all covariates included in adjusted models. This selection was based on a consensus decision-making process with health service and community partners, and informed by previous literature. ${ }^{15}$ Adjusted ORs (aORs) with 95\% CIs were calculated to identify independent covariates that remained associated with lower knowledge. Where a variable had $>5 \%$ missing data, a missing indicator covariate was created and included in the analyses.

For our secondary analysis, we assessed participants' knowledge of HIV/STI transmission alone with the same factors described earlier. The results of this are provided in the supplement (including online supplemental table 3).

A sensitivity analysis assessing low knowledge among only HIV-negative MSM was also conducted, with the results available in online supplemental table 4.

In addition, collinearity between independent covariates was tested. In the correlation matrices of predictor covariates, all pairwise Pearson correlation coefficients were $<0.4$, suggesting that collinearity was not an important factor in this study. All data analyses were undertaken in StataSE V.15.

\section{RESULTS}

The number of valid responses to MISI 2015 was 3090. We excluded 185 men who did not answer all 13 questions relating to HIV/STI treatment, transmission and testing, and our final sample was 2905 men (94\% of total). The median age was 30 years (range 18-78 years), with 5\% ( $\mathrm{n}=142)$ HIV positive. Ninety per cent $(\mathrm{n}=2611)$ of men had sex with another man within the previous 12 months. The median knowledge score for study participants was 11 out of 13 (IQR 10-13) (online supplemental table 2). A comparison between our study sample and the excluded men is shown in online supplemental table 5 .

\section{Lower knowledge}

One thousand and fifty-five men (36\%) had lower knowledge of HIV/STI testing, transmission and treatment. In univariable analysis, factors associated with having lower knowledge are shown in table 1 .

From the multivariable analysis, lower knowledge was significantly more likely among men aged $18-24$ years (aOR 1.98 , 95\% CI 1.50 to 2.61), those educated below degree level (aOR $1.58,95 \%$ CI 1.32 to 1.88 ), men living outside Dublin (aOR $1.21,95 \%$ CI 1.01 to 1.44 ), those born in Ireland (aOR 1.62, $95 \%$ CI 1.25 to 2.10 ) and among men who were unsure of their HIV status (aOR 1.35, 95\% CI 1.13 to 1.61). Lower levels of knowledge were also observed among men who were out to fewer people, with significantly higher aORs observed in those out to few (aOR 1.36, 95\% CI 1.06 to 1.76) or none (aOR 1.69 , $95 \%$ CI 1.20 to 2.37 ) compared with those out to all/almost all. MSM who had never tested for HIV also had lower knowledge levels compared with those who last tested negative for HIV within the previous 12 months (aOR 2.32, 95 CI 1.88 to 2.86). Furthermore, non-engagement with the national MSM-specific health promotion website was associated with lower knowledge (aOR 1.81, 95\% CI 1.45 to 2.25 ). Full results, including the baseline comparison group for each covariate, can be seen in table 1 .

Of note from the secondary analysis, in multivariable-adjusted models, students had low knowledge levels of HIV/STI transmission compared with men who were employed (aOR 1.35, 95\% CI 1.07 to 1.71 ). In addition, age, education level, country of birth, outness level, HIV testing history, certainty of HIV status and prior non-engagement with the national MSM-specific health promotion website were significantly associated with knowledge levels. In contrast to the primary analysis, men living outside of Dublin did not appear to have low knowledge of HIV transmission compared with men living in Dublin (aOR 1.04, $95 \%$ CI 0.88 to 1.23 ). The full results of the secondary analysis can be seen in the supplemental results section (online supplemental table 3).

\section{DISCUSSION \\ Principal findings}

Our primary analysis included 2905 MSM living in Ireland in 2015, before the widespread availability of PrEP. Although the majority of MSM in Ireland had high levels of knowledge about HIV/STI transmission, testing and treatment, a large minority $(36 \%)$ had low knowledge. While knowledge is only a single component of health literacy, its importance has been documented in prior studies which have shown that knowledge of HIV is a good predictor of a person's overall health literacy. ${ }^{12}$ Previous research has highlighted the benefits of interventions aimed at improving knowledge of HIV/STI transmission, testing and treatment among MSM, with some evidence of subsequent reductions in risky sexual behaviour. ${ }^{13}$

In total, $56 \%$ of MSM who had never tested for HIV had lower knowledge compared with only $9 \%$ of HIV-positive MSM. These results are similar to those from existing studies which reported that HIV-positive MSM tend to have better knowledge about HIV treatment and onward transmission risks, likely due to their HIV diagnosis acting as an educational intervention. ${ }^{14} \mathrm{~A}$ similar trend was observed in our secondary analysis, which focused on HIV/STI transmission only. Lack of knowledge about HIV 
Table 1 Univariable and multivariable analyses of lower knowledge of HIV and STI transmission, testing and treatment among MSM in Ireland, MISI 2015

\begin{tabular}{|c|c|c|c|c|c|c|c|}
\hline & \multirow{2}{*}{$\begin{array}{l}\text { Number } \\
\text { with lower } \\
\text { knowledge (\%) }\end{array}$} & \multicolumn{3}{|c|}{ Univariable analysis } & \multicolumn{3}{|c|}{ Multivariable analysis } \\
\hline & & OR & $95 \% \mathrm{Cl}$ & $P$ value & $\mathrm{aOR}$ & $95 \% \mathrm{Cl}$ & $P$ value \\
\hline \multicolumn{8}{|l|}{ Age group (years) } \\
\hline$>40$ & $234(30)$ & 1 & Ref & - & 1 & Ref & - \\
\hline $25-39$ & $353(29)$ & 0.98 & (0.80 to 1.19$)$ & 0.82 & 1.22 & (0.98 to 1.53$)$ & 0.080 \\
\hline $18-24$ & $468(51)$ & 2.48 & (2.03 to 3.02 ) & $<0.001$ & 1.98 & (1.50 to 2.61$)$ & $<0.001$ \\
\hline \multicolumn{8}{|l|}{ Education } \\
\hline Degree or higher & $431(28)$ & 1 & Ref & - & 1 & Ref & - \\
\hline Less than a degree & $601(46)$ & 2.20 & (1.88 to 2.57 ) & $<0.001$ & 1.58 & (1.32 to 1.88$)$ & $<0.001$ \\
\hline \multicolumn{8}{|l|}{ Area of residence ${ }^{*}$} \\
\hline Dublin & $403(30)$ & 1 & Ref & - & 1 & Ref & - \\
\hline Outside Dublin & $589(42)$ & 1.69 & (1.44 to 1.97$)$ & $<0.001$ & 1.21 & (1.01 to 1.44$)$ & 0.034 \\
\hline Missing & $63(34)$ & 1.20 & (0.86 to 1.66$)$ & 0.281 & 1.05 & (0.74 to 1.51$)$ & 0.771 \\
\hline \multicolumn{8}{|l|}{ Employment } \\
\hline Employed & $580(31)$ & 1 & Ref & - & 1 & Ref & - \\
\hline Unemployed & $83(44)$ & 1.74 & (1.29 to 2.36$)$ & $<0.001$ & 1.23 & (0.87 to 1.72$)$ & 0.237 \\
\hline Student & $322(49)$ & 2.18 & (1.82 to 2.62 ) & $<0.001$ & 1.10 & (0.86 to 1.41$)$ & 0.452 \\
\hline Othert & $49(40)$ & 1.51 & (1.04 to 2.19$)$ & 0.032 & 1.55 & (1.02 to 2.35$)$ & 0.039 \\
\hline \multicolumn{8}{|l|}{ Country of birth } \\
\hline Outside Ireland & $106(26)$ & 1 & Ref & - & 1 & Ref & - \\
\hline Ireland & $944(38)$ & 1.78 & (1.41 to 2.25 ) & $<0.001$ & 1.62 & (1.25 to 2.10$)$ & $<0.001$ \\
\hline \multicolumn{8}{|l|}{ Sexual Identity } \\
\hline Gay & $756(33)$ & 1 & Ref & - & 1 & Ref & - \\
\hline Bisexual & $185(48)$ & 1.81 & (1.45 to 2.24 ) & $<0.001$ & 1.04 & (0.80 to 1.37$)$ & 0.756 \\
\hline Straight/other & $98(45)$ & 1.64 & (1.24 to 2.17 ) & 0.001 & 0.90 & (0.65 to 1.25$)$ & 0.523 \\
\hline \multicolumn{8}{|l|}{ Outness level } \\
\hline Out to all/almost all & $433(30)$ & 1 & Ref & - & 1 & Ref & - \\
\hline More than half & $155(37)$ & 1.35 & (1.08 to 1.70$)$ & 0.009 & 1.16 & (0.90 to 1.48$)$ & 0.256 \\
\hline Less than half & $94(36)$ & 1.32 & (1.00 to 1.75$)$ & 0.047 & 1.03 & (0.76 to 1.41$)$ & 0.832 \\
\hline Few & $206(44)$ & 1.86 & (1.50 to 2.31$)$ & $<0.001$ & 1.36 & (1.06 to 1.76$)$ & 0.017 \\
\hline None & $136(54)$ & 2.70 & (2.06 to 3.54$)$ & $<0.001$ & 1.69 & (1.20 to 2.37 ) & 0.002 \\
\hline \multicolumn{8}{|l|}{ HIV testing history } \\
\hline Negative $<12$ months & $267(25)$ & 1 & Ref & - & 1 & Ref & - \\
\hline Negative $>12$ months & $169(29)$ & 1.23 & (0.98 to 1.54$)$ & 0.074 & 1.14 & (0.89 to 1.46$)$ & 0.285 \\
\hline Positive & $13(9)$ & 0.31 & (0.17 to 0.56$)$ & $<0.001$ & 0.38 & (0.21 to 0.69$)$ & 0.001 \\
\hline Never tested & $598(56)$ & 3.96 & (3.30 to 4.76 ) & $<0.001$ & 2.32 & (1.88 to 2.86$)$ & $<0.001$ \\
\hline \multicolumn{8}{|l|}{ STI in the last 12 months } \\
\hline No/don't know & $986(38)$ & 1 & Ref & - & 1 & Ref & - \\
\hline Yes & $62(23)$ & 0.48 & (0.36 to 0.65$)$ & $<0.001$ & 0.89 & (0.64 to 1.23 ) & 0.486 \\
\hline \multicolumn{8}{|l|}{ Certainty of HIV status } \\
\hline Sure of HIV status & $648(33)$ & 1 & Ref & - & 1 & Ref & - \\
\hline Unsure of HIV status & $400(43)$ & 1.50 & (1.28 to 1.76$)$ & $<0.001$ & 1.35 & (1.13 to 1.61$)$ & 0.001 \\
\hline Visited Man2Man website & & & & $<0.001$ & & & $<0.001$ \\
\hline Visited & $178(24)$ & 1 & Ref & - & 1 & Ref & - \\
\hline Not visited & $855(41)$ & 2.24 & (1.85 to 2.70$)$ & $<0.001$ & 1.81 & (1.45 to 2.25$)$ & $<0.001$ \\
\hline Viewed one or more HP infographics & & & & $<0.001$ & & & 0.022 \\
\hline Viewed & $465(32)$ & 1 & Ref & - & 1 & Ref & - \\
\hline Not viewed & $551(40)$ & 1.46 & (1.25 to 1.71$)$ & $<0.001$ & 1.17 & (0.98 to 1.41$)$ & 0.084 \\
\hline
\end{tabular}

*Variable with $>5 \%$ missing data.

tOther includes retired, sick leave, medically retired and other.

aOR, adjusted OR; HP, health promotion; MISI, MSM Internet Survey Ireland; MSM, men who have sex with men; Ref, reference.

transmission is associated with irregular condom use and higher numbers of sexual partners. ${ }^{15}$ This may contribute to potential undiagnosed HIV and STIs among this cohort of MSM. It is notable that over $25 \%$ of men who previously tested negative for HIV also had low knowledge levels. This is concerning as it appears that the opportunity for educating these men about HIV and STIs at the time of testing is being missed. It has been shown that MSM are likely to practice safer sex in the immediate aftermath of HIV testing and so perhaps may also be more receptive to long-term knowledge improving interventions at this time. ${ }^{21}$ 
Over one-third (37\%) of the sample had never tested for HIV and $32 \%$ disclosed that they were unsure of their HIV status. The proportion of men who had never tested for HIV was higher than reported in other recent surveys of MSM, and it may be partially explained by the relatively high response rates from younger MSM in MISI 2015. ${ }^{19}$ Based on the potential burden of undiagnosed HIV and STIs, non-testers and infrequent HIV testers are an important group to target for knowledge improvement. This requires interventions which increase awareness about the relevance of testing and which improve access to testing as a priority. A previous study highlighted the benefit of communitybased peer testing services for hard-to-reach groups like MSM, and similar interventions in an Irish setting may improve testing uptake among MSM. ${ }^{22}$

The lower levels of knowledge of HIV/STI transmission (from our secondary analysis) among students is a cause for concern. Heavy alcohol consumption in this group has been linked to inconsistent condom use previously. ${ }^{23}{ }^{24}$ It is plausible that lower levels of knowledge of HIV/STI transmission among students may also be contributing to high levels of risky sexual behaviour and onward spread of HIV and STIs. ${ }^{25}$ Improved knowledge about HIV transmission risk may improve condom use among students. ${ }^{26}$ The knowledge disparity among younger MSM also underlines the importance of providing interventions to educate younger MSM, and all students, on safer sexual practices in schools and third-level institutions. Multiple frameworks for how to develop and deliver such teaching have been produced previously and may be adapted to the Irish setting. ${ }^{27}$ These frameworks provide information for educators on how to maximise student participation in educational programmes and are associated with improved knowledge among young people.

MSM who disclosed their sexuality to few or no people had lower knowledge of HIV/STI transmission, testing and treatment when compared with MSM who were openly out to all or almost all. MSM who are out to more people are also more likely to disclose this fact to their medical practitioners and are more likely to seek information on topics relating to sexual health. ${ }^{28}$ While interventions could be tailored to MSM who are less likely to engage with sexual health services, interventions which aid MSM being more open about their sexual orientation are preferable. Stigma around LGBT+ issues still exists in Irish society and may negatively affect men's confidence in disclosing their sexual orientation..$^{29}$ It is thus important that emphasis is placed on support that is known to help improve 'outness', including mental health support, through the creation of LGBT+ social spaces. These interventions, in addition to the implementation of a National LGBT+ Inclusion Action Plan, may help accelerate acceptance of LGBT + issues nationwide and increase individuals' willingness to be open about their sexual orientation.

One potential option for reaching MSM is by making general health promotion materials accessible and appealing to larger audiences. Our findings show that a national health promotion website can be effective, perhaps more so than infographics or information leaflets in engaging MSM. Widespread promotion of health promotion materials, especially websites, may be beneficial for knowledge improvement, as research has shown that MSM may benefit from similar online interventions. ${ }^{13}$ However, it is important to consider the difficulties of evaluating the effectiveness of these materials and the potential limitations of selection bias among those who are likely to engage with such materials.

\section{Study limitations}

While MISI 2015 is currently the largest ever study of the MSM population in Ireland, it is not without limitations. First, we regret that we were unable to include a risk behaviour covariate (eg, number of sexual partners). However, this was unavoidable due to an intrinsic limitation in the way that data on the number of sexual partners were collected in MISI 2015. The learning from this survey has been used to inform the design of subsequent behavioural surveys of MSM in Ireland. In addition, all data were self-reported, with the survey only available online and through English. A convenience sample was used to maximise the size of the recruited sample. This may have introduced selection bias into the study, especially biassing the sample towards those who had computer access. However, previous research has highlighted that online surveys are the optimal method for recruiting a generalisable MSM sample, and so online recruitment is unlikely to have had major impact on the representativeness of our study population. ${ }^{30}$ Furthermore, we excluded participants who did not answer all 13 statements pertaining to HIV/STI knowledge. However, we compared the demographics of excluded participants with our study sample and found no major differences between the groups. We chose to dichotomise our outcome variable due to the skewed distribution of our outcome data, and based on consensus between the research team and community partners. This may have reduced some of the variability in the results, but it allowed for more meaningful and intuitive public health messages to be drawn from the analysis as it negated the need for a log (or other) transformation to be undertaken. Additionally, the cut-off values used for our lower knowledge variables were somewhat arbitrary and were chosen to ensure sufficient sample size in each group to detect possible true associations and to minimise the risk of a type II error rate. Finally, the survey was of cross-sectional design, and we cannot draw any temporal relationships between observed associations. We reported our findings in accordance with the Strengthening the Reporting of Observational studies in Epidemiology statement for cross-sectional studies (online supplemental file 2).

\section{CONCLUSION}

While the majority of MSM in Ireland were found to have high levels of knowledge about HIV/STI transmission, testing and treatment, our study highlighted that certain groups of MSM had knowledge deficits. We identified that lower knowledge was especially apparent among younger MSM, those MSM who had never tested for HIV and those MSM who were out to fewer people. These groups would likely benefit from targeted interventions to improve their knowledge of HIV and STIs. MSM who visited the national MSM-specific sexual health promotion

\section{Key messages}

- Large numbers of MSM in Ireland had poor knowledge regarding HIV and STI transmission, testing and treatment.

- Low knowledge was more apparent among younger MSM, those who had never tested for HIV, and among MSM who were out to fewer people.

- MSM who visited a national MSM-specific sexual health promotion website displayed higher knowledge levels of HIV and STI risks.

- MSM who have poorer knowledge of HIVISTI transmission pathways and testing opportunities may benefit from targeted interventions. 
website also had higher knowledge levels than those who did not.

Handling editor Jamie Scott Frankis

Acknowledgements The authors wish to thank all the men who gave their time to participate in MSM Internet Survey Ireland 2015. The authors acknowledge the support of partner organisations, including the Gay Men's Health Service, Gay Health Network, HSE Sexual Health and Crisis Pregnancy Programme, HIV Ireland, and all those who helped to promote the survey. We acknowledge in particular the help and support of Coralie Giese, Maeve O'Brien, Siobhan O'Dea, Marzena Sekular, Susan Donlon, Michelle Merrigan and Fiona Lyons.

Contributors CC, PB and DI designed the study. CC analysed the data under the supervision of $\mathrm{PB}$ and $\mathrm{DI}$. CC wrote the initial manuscript. All authors were involved in revising the manuscript for intellectual content, and all authors approved the final version of the manuscript.

Funding The authors have not declared a specific grant for this research from any funding agency in the public, commercial or not-for-profit sectors.

Competing interests None declared.

Patient consent for publication Not required.

Ethics approval Ethical approval was granted by the Royal College of Physicians in Ireland (ID: RECSAF30). All participants provided informed consent, and could withdraw from the study at any time.

Provenance and peer review Not commissioned; externally peer reviewed.

Data availability statement Data may be obtained from a third party and are not publicly available. Currently, the unpublished MISI data are available from the Health Protection Surveillance Centre in Dublin, Ireland. It is planned that the entire dataset will be uploaded to the Irish Social Science Data archive (https://www.ucd. ie/issda/) in the near future.

ORCID iD

Cian Carey http://orcid.org/0000-0002-9637-9603

\section{REFERENCES}

1 O'Leary D. The syndemic of AIDS and STDs among MSM. Linacre Q 2014:81:12-37.

2 HPSC. HIV in Ireland: 2018 provisional data including latest trends Dublin. Ireland: Health Protection Surveillance Centre, 2019.

3 HPSC. Sexually transmitted infections (STIS) in Ireland: 2018 provisional data Dublin. Ireland: Health Protection Surveillance Centre, 2019.

4 ECDC. Who regional office for Europe. HIVIAIDS surveillance in Europe 2018 2017 data. Copenhagen: European Centre for Disease Prevention and Control, 2019

5 CDC. Hiv/Aids testing Atlanta, GA: centers for disease control and prevention, 2018. Available: https://www.cdc.gov/hiv/basics/testing.html

6 Paz-Bailey G, Mendoza MCB, Finlayson T, et al. Trends in condom use among MSM in the United States: the role of antiretroviral therapy and seroadaptive strategies. AIDS 2016;30:1985-90

7 Glick SN, Morris M, Foxman B, et al. A comparison of sexual behavior patterns among men who have sex with men and heterosexual men and women. J Acquir Immune Defic Syndr 2012:60:83-90.

8 Shaw GM, Hunter E. HIV transmission. Cold Spring Harb Perspect Med 2012:2:a006965.
9 Hatzenbuehler ML, O'Cleirigh C, Mayer KH, et al. Prospective associations between HIV-related stigma, transmission risk behaviors, and adverse mental health outcomes in men who have sex with men. Ann Behav Med 2011;42:227-34.

10 Beyrer C, Baral SD, van Griensven F, et al. Global epidemiology of HIV infection in men who have sex with men. Lancet2012.

11 Carter A, Lachowsky N, Rich A, et al. Gay and bisexual men's awareness and knowledge of treatment as prevention: findings from the momentum health study in Vancouver, Canada. J Int AIDS Soc 2015:18:20039.

12 Hicks G, Barragan M, Franco-Paredes C, et al. Health literacy is a predictor of HIVIAIDS knowledge. Fam Med 2006;38:717-23.

13 Knight R, Karamouzian M, Salway T, et al. Online interventions to address HIV and other sexually transmitted and blood-borne infections among young gay, bisexual and other men who have sex with men: a systematic review. I Int AIDS Soc 2017;20:e25017

14 Mor Z, Turner D, Livnat Y, et al. HIV infected men who have sex with men in Israel: knowledge, attitudes and sexual behavior. BMC Infect Dis 2017;17:679.

15 Mor Z, Grayeb E, Beany A. Arab men who have sex with men in Israel: knowledge, attitudes and sexual practices. HIV Med 2016;17:298-304.

16 Gardner C, O'Donoghue R, Dermody A. Findings from the National HIV knowledge and attitudes survey 2017 and people living with HIV stigma survey Dublin. HIV in Ireland, 2017.

17 O'Donnell K, Fitzgerald M, Barrett P, et al. MISI 2015 findings from the men who have sex with men Internet survey. Dublin: Health Protection Surveillance Centre, 2016.

18 Barrett P, O'Donnell K, Fitzgerald M, et al. Drug use among men who have sex with men in Ireland: prevalence and associated factors from a national online survey. Int J Drug Policy 2019;64:5-12.

19 O'Donnell K, Fitzgerald M, Quinlan M, et al. Inequalities in HIV testing uptake and needs among men who have sex with men living in Ireland: findings from an Internet survey. HIV Med 2019;20:157-63.

20 Card KG, Armstrong HL, Lachowsky NJ, et al. Belief in treatment as prevention and its relationship to HIV status and behavioral risk. J Acquir Immune Defic Syndr 2018:77:8-16

21 Difranceisco W, Pinkerton SD, Dyatlov RV, et al. Evidence of a brief surge in safer sex practices after HIV testing among a sample of high-risk men and women. J Acquir Immune Defic Syndr 2005;39:606-12.

22 Mutch AJ, Lui C-W, Dean J, et al. Increasing HIV testing among hard-to-reach groups: examination of rapid, a community-based testing service in Queensland, Australia. BMC Health Serv Res 2017;17:310

23 Davoren MP, Shiely F, Byrne M, et al. Hazardous alcohol consumption among university students in Ireland: a cross-sectional study. BMJ Open 2015;5:e006045.

24 Scott-Sheldon LAJ, Carey MP, Carey KB. Alcohol and risky sexual behavior among heavy drinking college students. AIDS Behav 2010;14:845-53.

25 Mair C, Ponicki WR, Gruenewald PJ. Reducing risky sex among college students: prospects for context-specific interventions. AIDS Behav 2016;20 Suppl 1:109-18.

26 Randolph ME, Torres H, Gore-Felton C, et al. Alcohol use and sexual risk behavior among college students: understanding gender and ethnic differences. Am J Drug Alcohol Abuse 2009;35:80-4.

27 Aggleton P, Bhana D, Clarke DJ, et al. Hiv education: reflections on the past, priorities for the future. AIDS Educ Prev 2018;30:254-66.

28 Qiao S, Zhou G, Li X. Disclosure of same-sex behaviors to health-care providers and uptake of HIV testing for men who have sex with men: a systematic review. Am J Mens Health 2018;12:1197-214.

29 Higgins A, Doyle L, Downes C, et al. The LGBTIreland report: national study of the mental health and wellbeing of lesbian, gay, bisexual, transgender and intersex people in Ireland. Dublin, 2016

30 den Daas C, Doppen M, Schmidt AJ, et al. Determinants of never having tested for HIV among MSM in the Netherlands. BMJ Open 2016;6:e009480. 